\title{
Glucose-6-Phosphate Dehydrogenase of Trypanosomatids: Characterization, Target Validation, and Drug Discovery
}

\author{
Shreedhara Gupta, ${ }^{1,2}$ Mariana Igoillo-Esteve, ${ }^{1,3}$ Paul A. M. Michels, ${ }^{1,4}$ and Artur T. Cordeiro ${ }^{5}$ \\ ${ }^{1}$ Research Unit for Tropical Diseases, de Duve Institute, TROP 74.39, Avenue Hippocrate 74, 1200 Brussels, Belgium \\ ${ }^{2}$ Department of Chemistry, Heritage Institute of Technology, Chowbaga Road, Anandapur, Kolkata 700107, India \\ ${ }^{3}$ Laboratory of Experimental Medicine, Université Libre de Bruxelles, Route de Lennik 808, CP 618, 1070 Brussels, Belgium \\ ${ }^{4}$ Laboratory of Biochemistry, Université Catholique de Louvain, Brussels, Belgium \\ ${ }^{5}$ Laboratório Nacional de Biociências (LNBio), Centro Nacional de Pesquisas em Energia e Materiais (CNPEM), Caixa Postal 6192, \\ 13083-970 Campinas, SP, Brazil
}

Correspondence should be addressed to Paul A. M. Michels, paul.michels@uclouvain.be and Artur T. Cordeiro, artur.cordeiro@lnbio.org.br

Received 9 December 2010; Accepted 20 January 2011

Academic Editor: Hemanta K. Majumder

Copyright ( $) 2011$ Shreedhara Gupta et al. This is an open access article distributed under the Creative Commons Attribution License, which permits unrestricted use, distribution, and reproduction in any medium, provided the original work is properly cited.

In trypanosomatids, glucose-6-phosphate dehydrogenase (G6PDH), the first enzyme of the pentosephosphate pathway, is essential for the defense of the parasite against oxidative stress. Trypanosoma brucei, Trypanosoma cruzi, and Leishmania mexicana G6PDHs have been characterized. The parasites' G6PDHs contain a unique 37 amino acid long N-terminal extension that in T. cruzi seems to regulate the enzyme activity in a redox-state-dependent manner. T. brucei and T. cruzi G6PDHs, but not their Leishmania spp. counterpart, are inhibited, in an uncompetitive way, by steroids such as dehydroepiandrosterone and derivatives. The Trypanosoma enzymes are more susceptible to inhibition by these compounds than the human G6PDH. The steroids also effectively kill cultured trypanosomes but not Leishmania and are presently considered as promising leads for the development of new parasite-selective chemotherapeutic agents.

\section{Introduction}

The family Trypanosomatidae, belonging to the order Kinetoplastida, contains a large number of species, distributed over several genera. All known members of the Trypanosomatidae family are parasites, infectious to humans and other mammals, other vertebrates, insects and plants. The humaninfective trypanosomatids are grouped in species of two genera, Trypanosoma and Leishmania. They are responsible for a wide spectrum of diseases in tropical and subtropical countries. Two subspecies of Trypanosoma brucei, T. $b$. rhodesiense, and T. $b$. gambiense, are responsible for different forms of Human African Trypanosomiasis or sleeping sickness, an endemic disease in over 250 distinct foci in rural areas of 36 sub-Saharan African countries. The currently estimated number of cases is 50,000-70,000 with 17,000 new infections annually and 60 million people at risk $[1,2]$. The parasite is transmitted between human and/or other mammalian hosts by the tsetse fly. The complex life cycle of this extracellular parasite includes a procyclic form present in the midgut of the insect vector and a bloodstream form present in the blood of the mammalian hosts [3].

Trypanosoma cruzi is responsible for Chagas' disease in most countries of Latin America. It is estimated that 1118 million people are infected; 13,000 deaths are reported annually and about 100 million people are at risk [4]. The parasites are transmitted by blood-sucking reduviid bugs. After infection, the metacyclic trypomastigotes invade host cells where they proliferate as the so-called amastigote forms and, after differentiation into bloodstream trypomastigotes, they infect more cells, notably of heart muscle, and alimentary track tissue $[1,5,6]$.

Different species of Leishmania cause a variety of clinical symptoms, collectively called Leishmaniases. These diseases 
may involve cutaneous and mucocutaneous lesions, often causing severe debilitating wounds, or life-threatening visceral diseases in which vital organs are affected. The diseases threaten about 350 million people in 88 countries in tropical and subtropical parts of the world. An estimated 12 million people are currently infected with about 1-2 million new cases occurring annually. Leishmania transmission occurs via the byte of sandflies which inject metacyclic promastigote parasites into the skin. These forms enter macrophages where they reside as multiplying amastigotes within the phagolysosomes [7].

Sleeping sickness, Chagas' disease and visceral leishmaniasis can have a fatality rate as high as $100 \%$ if left untreated or not treated properly $[1,8]$. But treatment with drugs currently available is highly unsatisfactory [9, 10]. Most drugs have low efficacy and adverse side effects. Moreover the emergence of drug resistance is a continuous concern. Therefore, and because of the lack of efficacious vaccines, the discovery and development of effective drugs, nontoxic, affordable and easy to administer to the affected populations in the resource-poor areas is an urgent need.

Despite the tremendous progress made in recent decades in understanding the biochemistry and molecular biology of trypanosomatid parasites [11-14], chemotherapeutic treatment of the diseases has seen limited progress.

A currently common strategy for drug discovery against any parasitic diseases is to identify essential metabolic pathways associated with the parasites. In trypanosomatids, several enzymes involved in various metabolic processes have been characterized and established as promising drug targets [14, 15]. Among these validated targets is glucose6-phosphate dehydrogenase (G6PDH; EC 1.1.1.49), a key enzyme of the pentosephosphate pathway.

\section{The Role of the Pentosephosphate Pathway and Glucose-6-Phosphate Dehydrogenase}

In most organisms glucose is metabolized through two major pathways: the glycolytic and the pentosephosphate pathway (PPP) [16]. Whereas glycolysis serves for ATP production and to produce metabolites for use in a large variety of anabolic or further catabolic processes, the PPP can be divided in two successive phases with different functions. The first phase, called oxidative branch, generates reducing power under the form of NADPH and the second one, also known as nonoxidative or sugar interconversion branch, involves a series of reversible nonoxidative reactions leading to the conversion of the 5-carbon sugar resulting from the first phase into other metabolites (Figure 1). The oxidative branch comprises three enzymes: G6PDH, 6-phosphogluconolactonase, and 6-phosphogluconate dehydrogenase (6PGDH), whose successive activities convert glucose 6-phosphate (G6P) into ribulose 5-phosphate, with the concomitant production of NADPH by both dehydrogenases and $\mathrm{CO}_{2}$ release by the decarboxylation of the 6-phosphogluconate by 6PGDH [17]. The NADPH is used by the cell as a reducing agent in a variety of biosynthetic processes as well as for the defense against reactive oxygen species by keeping glutathione in its reduced state [18]. Glutathione is the major endogenous cellular antioxidant, participating directly in the neutralization of free radicals and reactive oxygen compounds, as well as maintaining exogenous antioxidants such as vitamins $\mathrm{C}$ and $\mathrm{E}$ in their reduced, active forms.

The nonoxidative branch comprises ribose-5-phosphate isomerase, ribulose-5-phosphate epimerase, transketolase and transaldolase, which, by isomerase and epimerase reactions and the transfer of two- and three-carbon units, form a variety of intermediates including ribose 5-phosphate, required for nucleotide synthesis, and erythrose 4-phosphate a precursor of aromatic amino acids and vitamins [19].

Depending on the cell's needs, the PPP can function partially or as a whole cycle by which one of the possible products, fructose 6-phosphate, is converted back into G6P, to enter the oxidative branch again. In addition, the PPP and glycolytic pathway are connected by sharing some intermediates such as G6P, fructose 6-phosphate and glyceraldehyde 3-phosphate.

G6PDH is the first committed enzyme of the PPP. Although most organisms have other means to produce cellular NADPH, notably by the enzymes isocitrate dehydrogenase and malic enzyme, the generally important role of G6PDH in it is illustrated by the severity of G6PDH deficiency in humans. The deficiency may lead to nonimmune hemolytic anemia triggered by oxidative stress as a result of infections or exposure to chemicals such as present in medication or certain foods [20]. G6PDH deficiency is the most common human enzyme defect, occurring in more than 400 million people worldwide [21].

\section{Glucose-6-Phosphate Dehydrogenase in Trypanosomatids}

The presence of G6PDH activity in trypanosomatids was first demonstrated in 1959 by Raw in T. cruzi epimastigotes [22] and in 1962 by Ryley in both cultured procyclic and bloodstream form T. $b$. rhodesiense [23]. A few years later, studies with labeled glucose by Mancilla and colleagues [24-26] suggested that the PPP is functional in some T. cruzi strains and Leishmania species and, much later, Voorheis and coworkers [27] confirmed the presence of a classical PPP in T. brucei by determining the specific activities of all its enzymes in both procyclic and bloodstream forms. Interestingly, no activities of ribulose-5-phosphate epimerase and transketolase were detectable in the bloodstream form and only low activity was found for transaldolase, suggesting an important differential repression of the nonoxidative branch in this lifecycle stage [27]. For some of the PPP enzymes, like G6PDH and 6-phosphogluconolactonase, a dual subcellular distribution was later found in both bloodstream and procyclic form trypanosomes. While their main enzymatic activity was present in the cytosol, approximately 40 and $10 \%$, respectively, of their activity was associated with glycosomes $[28,29]$. Glycosomes are the peroxisome-like organelles in Kinetoplastida which uniquely harbour the majority of the enzymes of the glycolytic pathway [30]. These organelles, 


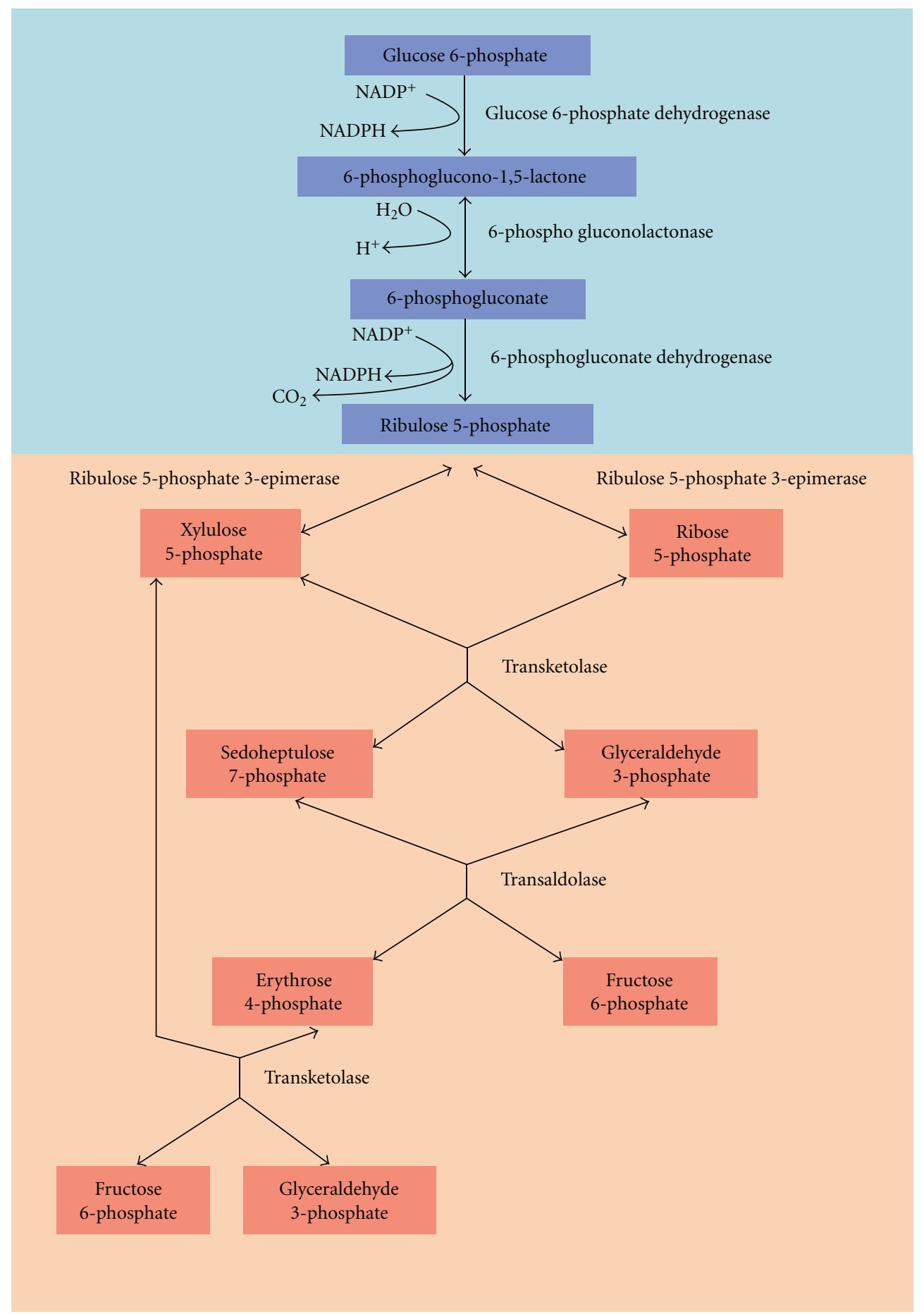

FIGURE 1: Schematic representation of the pentosephosphate pathway.

which number between 5 and 10 in Leishmania amastigotes and approximately 65 in bloodstream form T. brucei, may contain enzymes from a variety of different pathways [3133 , with the glycolytic enzymes being most notable and comprising even up to $90 \%$ of the organelles' protein content as observed in bloodstream form trypanosomes [33-35].

A functional PPP has also been unambiguously demonstrated in T. cruzi epimastigotes by measuring the $\mathrm{CO}_{2}$ production from radiolabeled glucose [36]. Moreover, the importance of the PPP was demonstrated by a challenge with methylene blue, mimicking oxidative stress, which caused a twofold increase of the flux through the PPP. All enzymes of the pathway were identified by activity measurements in the four major developmental stages of this parasite. The activities were shown to be predominantly cytosolic, with a possible small glycosomal component for most of them. 
TABle 1: T. brucei (Tb), T. cruzi (Tc), and L. mexicana (Lm) G6PDH kinetic constants for an ordered bi-bi-reaction mechanism.

\begin{tabular}{lcccrc}
\hline Source & $\begin{array}{c}K_{\mathrm{G} 6 \mathrm{P}} \\
(\mu \mathrm{M})\end{array}$ & $\begin{array}{c}K_{\mathrm{NADP}^{+}} \\
(\mu \mathrm{M})\end{array}$ & $\begin{array}{c}\mathrm{V}_{1} \\
(\text { nmoles of NADPH·s }\end{array}$ & $\begin{array}{c}\left.k_{\text {cat }}\right) \\
\left(\mathrm{s}^{-1}\right)\end{array}$ & $\begin{array}{c}K_{\mathrm{iG} 6 \mathrm{P}} \\
(\mu \mathrm{M})\end{array}$ \\
\hline $\mathrm{Tb}$ & $57.8 \pm 2.4$ & $9.4 \pm 0.4$ & $36.2 \pm 1.5$ & $16.4 \pm 0.6$ & $47.6 \pm 1.9$ \\
$\mathrm{Tc}$ & $206.0 \pm 4.2$ & $22.5 \pm 1.2$ & $77.7 \pm 2.5$ & $57.1 \pm 1.9$ & $105.3 \pm 4.6$ \\
$\mathrm{Lm}$ & $74.5 \pm 3.0$ & $12.1 \pm 0.5$ & $31.2 \pm 1.2$ & $22.2 \pm 0.9$ & $86.4 \pm 3.5$ \\
\hline
\end{tabular}

Data from Cordeiro et al. $[45,46]$.

In a comparable study, the presence of a functional PPP was also demonstrated in promastigotes of Leishmania mexicana, by its twofold stimulation by methylene blue. Each of the classical PPP enzymes was identified and its specific activity measured [37]. Furthermore, it was shown that glucose serves as a source for nucleic acid synthesis, an indication that, in these parasites, the PPP plays also a role in the conversion of glucose into ribose 5-phosphate for nucleotide biosynthesis. Also in Leishmania, the PPP has probably a dual subcellular localization, although it has only been demonstrated so far for the transketolase that is predominantly present in the cytosol of promastigotes, but also with a significant component associated with the glycosomes [38]. The presence of the PPP in glycosomes of the three trypanosomatid species is further supported by the presence of peroxisomal-targeting signals (PTSs) in a number of enzymes of both the oxidative and nonoxidative branches [31, 39] and-for T. brucei-by the results of proteomic analyses $[31,32]$.

Whereas in almost all organisms, glutathione, reduced by NADPH produced predominantly in the PPP, is the major intermediate in the defense against reactive oxygen species, in trypanosomatids a different thiol redox component is being used, trypanothione, or $\mathrm{N}^{1}, \mathrm{~N}^{8}$-bis-gluthionyl-spermidine. Several enzymes involved in trypanothione metabolism have been detected both in the cytosol and glycosomes [31].

Preliminary enzymatic studies on G6PDH have been performed with the partially purified enzyme from T. cruzi $[40,41]$ and T. brucei [28], but detailed characterization of G6PDH of each of the three trypanosomatid species was only performed after the genes had been identified and used to produce the recombinant proteins. Genomic analysis revealed one gene copy per haploid genome in T. brucei [29] and L. mexicana [42] and several genes located on three different chromosomes of T. cruzi clone CL Brener $[43,44]$. Two of them were classified as pseudogenes, while the others were clustered in three groups of nearly identical $(98 \%)$ coding regions but with considerably different noncoding flanking sequences. The amino acid sequences of the functional G6PDHs of three trypanosomatids are 64 to $69 \%$ identical and share about 50\% identity with the human enzyme.

The T. brucei and T. cruzi G6PDH gene sequences both have two possible start codons, $111 \mathrm{bp}$ apart (Figure 2), while in Leishmania species only a unique start codon was found corresponding to the first one of the Trypanosoma genes. The region between the two start codons codes for a usual 37 amino acid N-terminal extension only present in the trypanosomatid G6PDHs. The long form of the $T$. brucei and T. cruzi enzymes code for polypeptides of 557 and 555 residues, respectively, while the L. mexicana G6PDH polypeptide encompasses 562 residues. Western blot analysis, performed with an antiserum raised against the N-terminal peptide of the T. cruzi G6PDH, showed that mainly the long form of the enzyme is expressed in all four life-cycle stages of this parasite [43]. Whether the long form of T. brucei G6PDH is also produced remains to be determined. Duffieux and coworkers [29] proposed that an ORF starting at the first ATG codon would lead to an mRNA with an unusually short $5^{\prime}$ untranslated region.

The L. mexicana G6PDH, the short form of the $T$. brucei enzyme and both the long and short form of $T$. cruzi G6PDH have been expressed with an N-terminal Histag in Escherichia coli, purified and kinetically characterized [29, 43, 45, 46]. The kinetic mechanism has been studied, under conditions which were similar for all enzymes, and appeared to follow for each of them a bi-bi-ordered pattern $[45,46]$. The values of the kinetic constants are presented in Table 1.

Interestingly, it was shown that the long and short form of T. cruzi G6PDH present several differences [43]. Experiments performed independently from those for which the results are presented in Table 1, revealed differences in the apparent $K_{m}$ values for G6P. While the purified recombinant long and short T. cruzi G6PDH had apparent $K_{m}$ values of 189.9 and $98.4 \mu \mathrm{M}$, respectively, that of the partially purified enzyme from parasites was $288 \mu \mathrm{M}$. In contrast, the apparent $K_{m}$ values for $\mathrm{NADP}^{+}$did not differ. Additionally, both the natural enzyme and the long recombinant G6PDH, but not the short form, were inactivated by reducing agents such as dithiothreitol, $\beta$-mercaptoethanol, and reduced glutathione. This effect, reminiscent to the redox-state-dependent activity regulation observed for G6PDH and some other enzymes from chloroplasts and cyanobacteria [47], was attributed to the presence of two Cys residues in the N-terminal peptide [43]. The corresponding N-terminal region of G6PDH of Leishmania spp. and the possible extension of the T. brucei enzyme do not contain these Cys residues (Figure 2). These observations suggest that among the trypanosomatid G6PDHs studied, only the activity of the T. cruzi enzyme is regulated by the redox state of the cell, in a similar way as reported for the enzyme from chloroplasts and cyanobacteria. Indeed, kinetic studies with the two purified recombinant forms showed that the long form was 4 -fold more sensitive for inhibition by the product NAPDH than the short one, suggesting that its activity is regulated by 


\begin{tabular}{|c|c|c|}
\hline ruzi & 1 & MSGS . . ENRCRFQKEVDKFFDTL RERTLRDH QELCEYLMENAKKVAAELRGEVCERIP \\
\hline T. brucei & 1 & NDGD . . LSOLGFVQEV DFFDVL REKTLRDR DNIPEYVMONCRKVADTLRKDS CDIIP \\
\hline mexicana & 1 & USEEQFAASOEAYVADVDSIL VL AQVLERK DNVLQFISKSALSLQKDSSSES CDLIS \\
\hline$a n$ & 1 & MAEQ $\ldots \ldots \ldots$ VALSRTQVCGILREELFQG . . . . . . . . . \\
\hline T. cruzi & 58 & DAVSPELRSRALIVVL $\overline{\text { GASGLA }}$ KTF ALF QLYCNGML RDV ILGYA $\stackrel{\#}{ }$ STMEDVEK \\
\hline T. brucei & 58 & DAAAPELKE ALT IVVLGA G LARN TTF ALFQLF CNGLI RTI IVGYA TKMPDVEQ \\
\hline mexicana & 61 & CKVKDEQKSR SLIIVFGASGLA KKTF ALF SLYCGGLL PDV IIGYA TKVDAEK \\
\hline Human & 24 & ... DAFHQ $\triangle$ DTH IF I IMGA SG LA KKK IY T IWWLFRDGLL ENTF IVGYA SRLTVADI \\
\hline T. cruzi & 8 & W: KDTLAGFFTRLDER GC VGNFLRRI YYMG YDRDEDFA L ERILRM EAFQGPE G \\
\hline T. brucei & 18 & W:KESLAKHFPRAKD CP IEAFLKTITYISG $Y$ Y GADDFF $L$ DVITKF ESFPGKQ G \\
\hline mexicana & 21 & W HETLMKHF SNLSEREC AE DFLKHI YFCG Y YKVEDFK LDIMIREK NAFKGPE G \\
\hline Human & 15 & Q QS . . EPFFKATPEEKLKL DFFARN $Y Y$ VAGQY DDAASYQ L SHMN . . ALHLGSQ . . \\
\hline T. cruzi & 178 & G RLFYLAL P SVFVGVCRGLSKGA QKPELGWV LIVEKPG RDTETSEQL NOLK LF \\
\hline T. brucei & 178 & G RLFYLAL PSVF MHACTGIRTHV QKPGLGWV IIIEKPFG TIES SNEL SROLE PF \\
\hline mexicana & 181 & G RLFYLAL PPVFATVCESIYKGVM PQEVGGWV VII EKPFG RDIE S SAEL RALE PFF \\
\hline Human & 134 & A RLYLAL P TVYEAVTKNIHESC SQ . IGWN IIVEKPG RDL. SDRL NHISSLF \\
\hline T. cruzi & 238 & 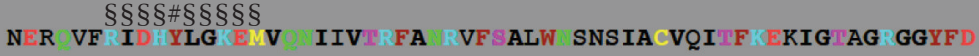 \\
\hline T. brucei & 238 & EES IFRIDHYG KEMV IVVIRFA RVFSALW NN IACVRITFKESIGIEG GGYFD \\
\hline mexicana & 241 & NES IYRIDYLG KMV IITIRFA RIFSAVW SS IACVQITFKETIGTEGGGYD \\
\hline Human & 191 & RED IYRI YLG KMV LMVLRA RIFGPIW RD IACVILTFKEPFGIEG GGYFD \\
\hline T. cruzi & 298 & SIGII RDVI LT ILSLLTMEKPRSLSAEDI RDEK VQVLRQVV ANPAECVLG YTAS \\
\hline T. brucei & 298 & KAGI I RDVV HT ILSLLAMEKPRSLSPED I RDE VIVLRHVN VTPADCVLG YTRS \\
\hline mexicana & 301 & SIGI I RDVM LT ILALL. MEKPRTLDAECI RDEK VSVLKHIE VTKENCVLG YIAS \\
\hline Human & 251 & EFGII RDVM LL MLCLVAMEKPASTNSD VRDEK VKVLKCISEVQANNVVLG YVGN \\
\hline T. cruzi & 358 & ADG... ST GYLDD SV KGSHCPIFAVL LHV \\
\hline T. brucei & 358 & EDG...SI GYLEDPIV RGSKCATFVVL LFI \\
\hline mexicana & 360 & ADG...SI GYLEDEIV GSTCPIFAVM LNI DRWAGV FILKAG AVE QKYVAI I \\
\hline Human & 311 & PDGEGEATKGYLDD PIV RGSTTA FAAVVLYVE E RWDV FILRCG ALNE KAEV L \\
\hline T. cruzi & 415 & FKDEIR. PFGESTQR ELVIRA PSEAYYL LTA T GL...LNDTH TELLYYRR \\
\hline T. brucei & 415 & FKDEIR. PFGVAAQR ELII A PSEAMYLRLA T GV...LSDTH TELLSYEHRY \\
\hline mexicana & 416 & FKDEVH. PYGE...R ELVI A PSEAYYVITT V GLSGDLRQTH TELLYHTY \\
\hline Human & 371 & FHDVAGDIFHQQCK R ELVI $: V$ N AVYT MMT K GM . . FFNPEES LDLYGN RY \\
\hline T. cruzi & 471 & D. VTL AYESLIHEALLGNST FV VDELAAWR IYT LL AID GEV VL P YAAGSCG \\
\hline T. brucei & 471 & N.ITL DAYESLIHEALLGRST FV KDELAAWR IYT LLEAIE GETTTY PYSAGSKG \\
\hline mexicana & 472 & D. VRL DAYESLLNDALLGSST FV KDELVAWRIFT LL QID GEI PIQYQAGTRG \\
\hline Human & 428 & KNVKL DAYERLILDVFCGSOMHFV SDELREAWR IFT LL QIELEKP PI PYIYGSRG \\
\hline T. cruzi & 530 & EEAQEF IRISGY TTNGNAYKCSNA \\
\hline ei & 530 & A:AQKFVDDTGF PLTGDIYQQRKLHHL \\
\hline mexi & 531 & KEADEF IISNGF KOKGYHWLPFNKL \\
\hline Human & 488 & JGFQYEGTYKWVNPHKL \\
\hline
\end{tabular}

FIGURE 2: G6PDH amino acid sequence alignment. The predicted amino acid sequences of T. cruzi G6PDH-long (DQ408239, sharing the highest identity with its T. brucei counterpart), T. brucei G6PDH-long (CAC07816), L. mexicana G6PDH (AAO37825), and Human G6PDH (AAL27011) were aligned using the CLUSTALW software. The first and second candidate initiator methionines in the T. cruzi and T. brucei sequences are indicated with arrows. The two regulatory cysteines of the T. cruzi G6PDH are boxed in yellow. Overlined, cofactor binding site; ${ }^{\S} \mathrm{G} 6 \mathrm{PDH}$ signature (residues belonging to the substrate binding site); ${ }^{\#}$ residues involved in substrate and cofactor binding.

the $\mathrm{NADP}^{+} / \mathrm{NADPH}$ ratio in the cell compartment where the enzyme is located, similarly as has been proposed for the enzyme from Anacystis nidulans [48].

The importance of G6PDH in the defense against oxidative stress has been demonstrated for both the T. cruzi [43] and T. brucei enzyme (SG and PM, unpublished; see below). Incubation of $T$. cruzi with $\mathrm{H}_{2} \mathrm{O}_{2}$ modified the G6PDH expression and specific activity in the cell (Figure 3). In metacyclic trypomastigotes, the life-cycle stage naturally exposed to reactive oxygen species (ROS) produced by the mammalian host, the peroxide exposure enhanced the G6PDH expression as well as the specific activity of the 

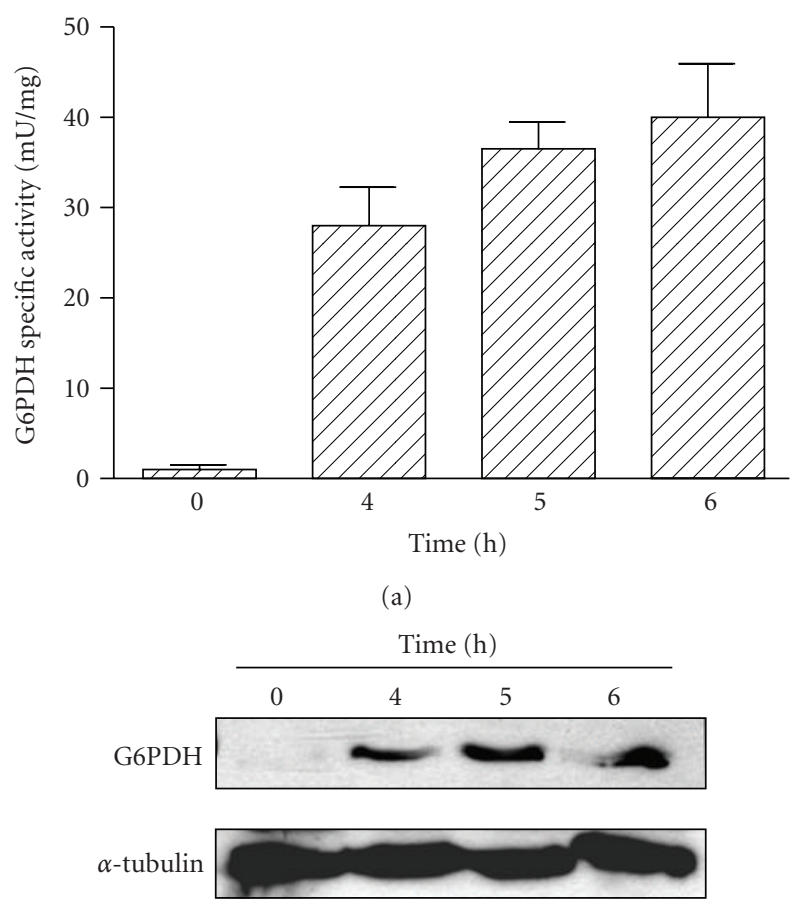

(b)

Figure 3: T. cruzi G6PDH expression and specific activity are enhanced by oxidative stress in metacyclic trypomastigotes. Parasites were incubated for 4 to $6 \mathrm{~h}$ in the presence of $70 \mu \mathrm{M} \mathrm{H}_{2} \mathrm{O}_{2}$. Samples for enzymatic activity measurements and western blots were taken before (T0) and after the $\mathrm{H}_{2} \mathrm{O}_{2}$ addition. (a) G6PDH specific activity. The results are means \pm SE of quadruplicates. (b) Autoradiography of a western blot corresponding to metacyclic trypomastigotes cell-free extracts ( $30 \mu \mathrm{g}$ of total protein/lane). $\alpha$ Tubulin was used as loading control. (Figure created with data from [43].)

enzyme. Conversely, peroxide treatment in epimastigotes, a life-cycle stage not naturally encountering such a host response, repressed the G6PDH expression and activity. In bloodstream forms of T. brucei G6PDH expression has been knocked down by tetracycline-inducible RNA interference (RNAi) [45]. Cells partially depleted from G6PDH showed enhanced sensitivity to different concentrations of $\mathrm{H}_{2} \mathrm{O}_{2}$ compared to wild-type trypanosomes (Figure 4).

\section{Glucose-6-Phosphate Dehydrogenase Is a Genetically Validated Drug Target in Bloodstream Form T. brucei}

As stated before, most organisms can produce NADPH not only by the action of the two dehydrogenases of the PPPG6PDH and 6PGDH-but also via isocitrate dehydrogenase and malate dehydrogenase to cope with G6PDH deficiencies. However, trypanosomatids depend only upon malic enzyme [49] and G6PDH for their cytosolic NADPH production. In these parasites the isocitrate dehydrogenase is compartmentalized inside the mitochondrion and, in species of
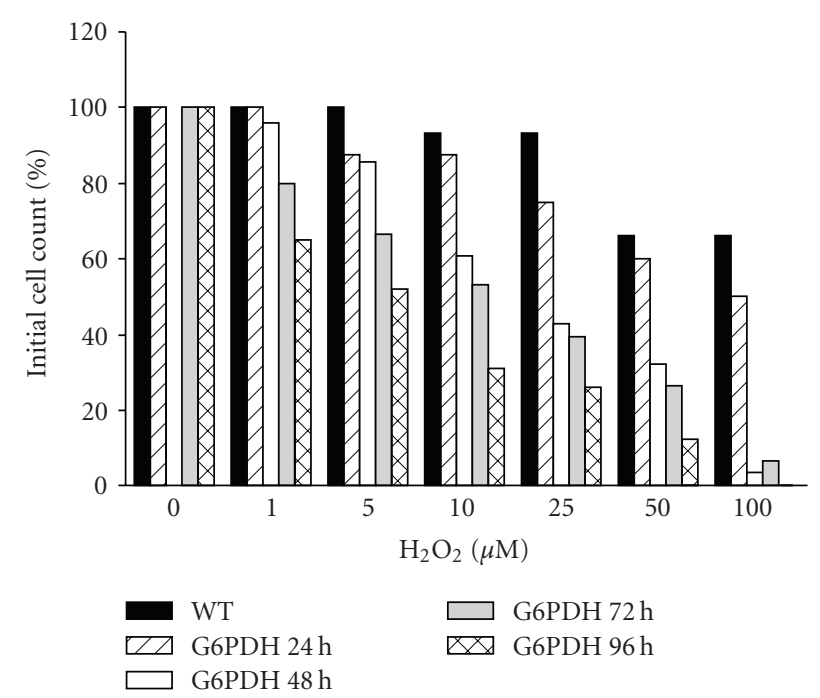

FIGURE 4: G6PDH is important for defense against oxidative stress in bloodstream form T. brucei. Wild-type (WT) trypanosomes and cells in which partial depletion of G6PDH was induced by RNAi were grown in regular HMI-9 medium. After growth for different periods of time as indicated, cells were collected by centrifugation and resuspended in non-reducing medium (i.e., without $\beta$-mercaptoethanol and cysteine) and the cell density was determined. Cell suspensions were then incubated for $1.5 \mathrm{~h}$ in the presence of different concentrations of $\mathrm{H}_{2} \mathrm{O}_{2}$ after which the cell density was determined again.

Trypanosoma but not Leishmania, it has also been predicted as a glycosomal enzyme [39].

As mentioned above, T. brucei bloodstream form G6PDH RNAi cell lines have been created [45]. To determine if inhibition of G6PDH (and consequently the flux through the downstream located PPP enzyme 6PGDH) was sufficient to affect the trypanosome growth, a cell line in which RNAi induction led to a considerable reduction in G6PDH expression was studied. In these cells $24 \mathrm{~h}$ of RNAi induction led to a mild decrease in G6PDH, while $48 \mathrm{~h}$ of knockdown induced a substantial reduction in $\mathrm{G} 6 \mathrm{PDH}$ protein, as determined by western blot analysis. The decrease in G6PDH expression was paralleled by an impairment in the trypanosomes' growth rate. This effect was observed between 24 and $48 \mathrm{~h}$ after the addition of tetracycline to the culture medium. Beyond $48 \mathrm{~h}$ of RNAi induction, G6PDH depleted cells started to die (Figure 5). Parasites containing the RNAi construct, but grown in the absence of tetracycline, showed a similar growth rate as wild-type cells grown in the presence or absence of tetracycline. The decrease of the growth rate correlated with the initiation of the RNAi induction and a simultaneous decrease in the protein level, suggesting that G6PDH plays an important role in growth and survival of the bloodstream form trypanosomes. The possibility that the observed phenotype was a consequence of an RNAi-offtarget effect cannot be excluded, but it was considered to be unlikely since the G6PDH sequence is unique and no other highly similar gene was identified in the T. brucei genome database. 


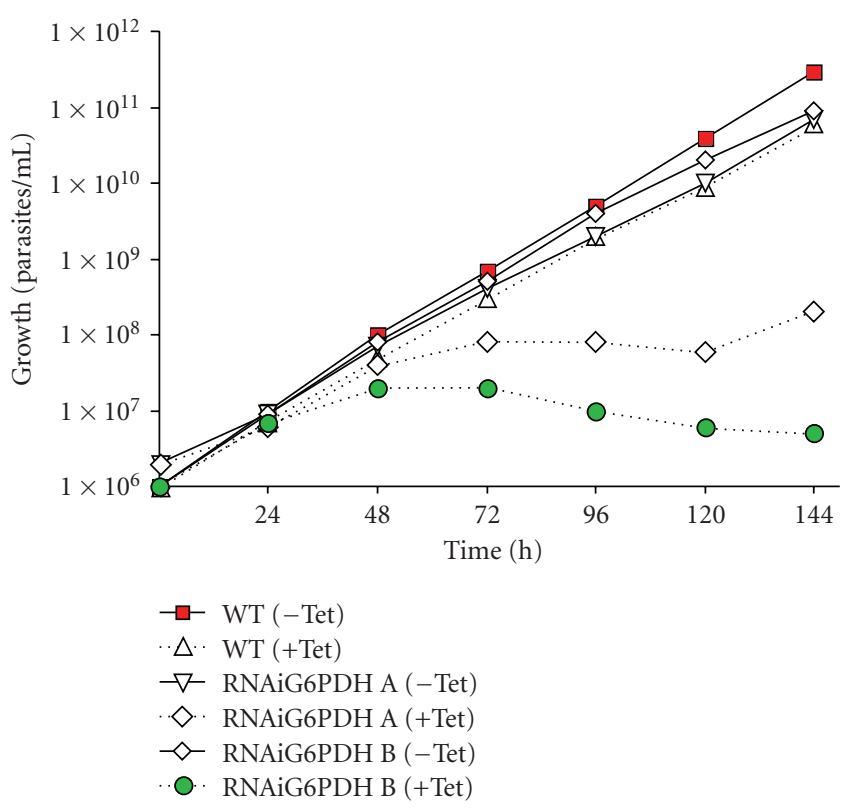

Figure 5: Genetic validation of G6PDH as a drug target in bloodstream form T. brucei. Cumulative growth of wild-type (WT) trypanosomes and two independent cell lines (RNAiG6PDH A and $\mathrm{B}$ ). In the absence of RNAi induction (-Tet), WT and RNAi parasites grow at equal rates; in the presence of the RNAi inducer tetracycline (+Tet), WT cells grow at a normal rate, while trypanosomes of the RNAi-cell lines die after $48 \mathrm{~h}$. (Figure created with data from [45].)

These RNAi experiments genetically validated G6PDH as a drug target in bloodstream forms of T. brucei and suggested that the NADPH produced by other enzymes than G6PDH is not sufficient to deal with oxidative stress experienced during normal or stress conditions (Figure 4).

\section{Inhibition of Trypanosomatid G6PDH by Steroids}

Inhibition of mammalian G6PDH by intermediates of the steroid-hormones biosynthesis was discovered 50 years ago by Mark and Banks [50]. They demonstrated a highly specific and uncompetitive inhibitory effect of dehydroepiandrosterone (DHEA) and derivatives on human G6PDH. These steroid molecules did not inhibit the homologous enzyme of either yeast or spinach. Moreover they did not affect the activity of mammalian 6PGDH and isocitrate dehydrogenase. During the following 35 years, it was a generally accepted notion that only G6PDH from mammalian cells was inhibited by steroid hormone derivatives [51]. As a consequence of such a generalization, the inhibition of G6PDH from lower eukaryotes, like trypanosomatids and other human parasites, was neglected. Observations that DHEA or derivatives of it were effective against infections of Cryptosporidium parvum [52], Plasmodium species [53], Schistosoma mansoni [54], and T. cruzi [55] were attributed to a stimulation of the host's immune response by the steroids. Nonetheless, in recent years it was shown that the steroids also affect growth of cultured parasites such as Entamoeba histolytica [56] and Taenia crassiceps [57], suggesting that they could have a direct effect on these organisms.

Recently, Cordeiro et al. [45] confirmed these findings by showing that steroids kill in vitro grown T. brucei bloodstream forms with $\mathrm{ED}_{50}$ values in the micromolar range. They additionally demonstrated that T. brucei G6PDH, the first nonmammalian enzyme, was effectively inhibited by DHEA and epiandrosterone (EA). Similar observations were subsequently made for T. cruzi G6PDH [46]. Although, DHEA itself failed to decrease the growth rate of cultured T. cruzi, its brominated derivatives 16BrDHEA and 16BrEA (Figure 6), which are more potent inhibitors of G6PDHs, presented $\mathrm{ED}_{50}$ values in the micromolar range, comparable to values reported for benznidazole [58], the currently available drug in use for treatment of Chagas' disease. Curiously, DHEA and EA did not inhibit L. mexicana G6PDH and had no effect on the growth rate of cultured promastigote parasites. This observation was indirect evidence for the specific inhibition of G6PDH by DHEA in trypanosomes.

\section{G6PDH Is the In Situ Target of Human Steroids with Trypanocidal Action}

Recently, the mechanism by which DHEA and EA kill $T$. brucei bloodstream forms has been evaluated, by exploiting the fact that these compounds showed no inhibitory effect on the recombinant L. mexicana G6PDH. Inhibitors were tested on the cell growth of a trypanosome clone that has been created to also express a transgene encoding this L. mexicana enzyme [59]. Whereas wild-type bloodstream form T. brucei showed a dose-dependent killing by DHEA and EA with $\mathrm{ED}_{50}$ values of $41.8 \pm 2.1 \mu \mathrm{M}$ and $21.4 \pm 1.6 \mu \mathrm{M}$, respectively, the T. brucei ( $\mathrm{LmG6PDH)}$ transgenic parasites showed no growth inhibition whatsoever by the two compounds, even at concentrations up to $100 \mu \mathrm{M}$ [59]. Thus, transfection of T. brucei bloodstream form parasites with $L m \mathrm{G} 6 \mathrm{PDH}$ could rescue the trypanosomes from being killed by DHEA and EA. This result confirms that the toxic effect of DHEA and EA on the parasites is uniquely due to the inhibition of their G6PDH.

\section{Conclusions and Perspectives}

Our research has validated the key PPP enzyme G6PDH as a target for new drugs to be developed against trypanosomes. Oxidative insults induce G6PDH expression and enhance its specific activity, while the partial depletion of this enzyme by RNAi makes the parasites vulnerable to oxidative stress. Prolonged depletion of G6PDH from cultured bloodstream form T. brucei resulted even in death of the parasites. Whether this was only due to an effect on the redox metabolism or also on the synthesis of metabolic intermediates to be used for biosynthetic processes still needs to be addressed. 


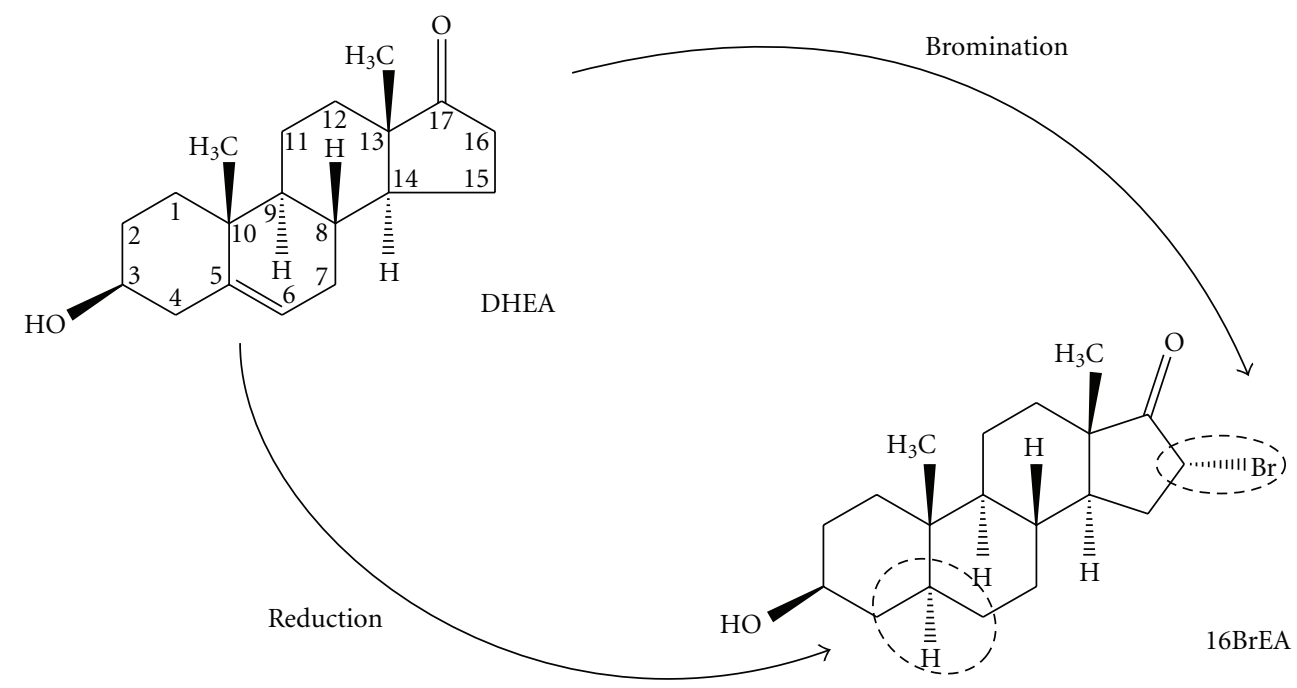

Figure 6: Steroid inhibitors of Trypanosoma G6PDH. Two modifications used to increase the inhibitory potency of steroids to G6PDH are indicated: reduction of the double bond between carbons 5 and 6 in dehydroepiandrosterone (DHEA) which leads to epiandrosterone (EA) and the bromination at position 16 which converts DHEA and EA into 16BrDHEA and 16BrEA, respectively.

Steroids derived from DHEA are potent uncompetitive inhibitors of Trypanosoma G6PDH, and indeed kill the parasites by in situ inactivating this enzyme. The uncompetitive nature of this inhibition is particularly relevant. Contrary to the much more often observed competitive inhibition, the increase of substrate concentration, resulting from this process, will not overcome the inhibition but may rather lead to an increase of metabolic intermediates to toxic levels [60]. The molecular mechanism by which the steroids inhibit the G6PDH of human, Trypanosoma and other lower eukaryotes including several human parasites, while they do not affect the activity of the enzyme from plants, yeasts, and Leishmania is not yet known. To analyze the differences between the Trypanosoma and human G6PDH's steroid binding site and the binding mechanism of these molecules, cocrystallization of the enzymes with steroids and the determination of their crystal structure is needed. To date, no three-dimensional structure of a trypanosomatid G6PDH is available, but crystallization studies are presently ongoing and, when successful, may open new possibilities for the design and synthesis of a different class of molecules with even higher inhibitory potency and selectivity for the parasite G6PDH.

Since these steroids inhibit Trypanosoma G6PDHs at much lower concentrations than the mammalian G6PDH, they are promising leads for the development of new drugs for treatment of African sleeping sickness and Chagas' disease. The next steps in the development of drug candidates against these diseases will involve the selection of compounds with potent and highly selective inhibitory activity on cultured parasites versus human cells, and the evaluation of their efficacy, bioavailability, and toxicity in infected animal models.

The lack of inhibition of the Leishmania G6PDH by DHEA and its derivatives is puzzling. It is feasible that this is due to a single but crucial substitution in the enzyme that prevents the binding of these compounds. Screening of a steroid library against the available recombinant $L$. mexicana G6PDH may help to identify potent uncompetitive inhibitors with in vitro anti-Leishmania spp. activity.

As mentioned above, steroids also impaired the growth of other parasites, that is, Taenia crassiceps and Entamoeba histolytica, as well as the parasitaemia of Plasmodium falciparum and P. berghei, Cryptosporidium parvum, and Schistosoma mansoni. It is still necessary to determine if G6PDH is also in these cases the main target of the steroid molecules. This will open new perspectives for discovery of drugs also against the diseases caused by these parasites.

Concerning the trypanosomatid-borne diseases, the availability of recombinant G6PDH for each of the three parasites makes also possible an alternative strategy, that is, using these enzymes for high-throughput screening of large libraries of drug-like compounds. This approach may lead to very wide range of inhibitors potentially exploitable for antiparasitic treatment.

\section{Abbreviations}

G6P: $\quad$ Glucose 6-phosphate

G6PDH: Glucose-6-phosphate dehydrogenase

6PGDH: 6-phosphogluconate dehydrogenase

PPP: Pentosephosphate pathway

PTS: $\quad$ Peroxisome-targeting signal

RNAi: RNA interference

ROS: Reactive oxygen species.

\section{Acknowledgments}

This research was supported through grants to PAMM from the "Fonds de la Recherche Scientifique" (F.R.SFNRS) of the "Communauté Française de Belgique" and the Interuniversity Attraction Poles-Belgian Federal Office 
for Scientific, Technical and Cultural Affairs. S. Gupta, M. Igoillo-Esteve and A. Cordeiro each gratefully acknowledge the "de Duve Institute" for having previously been awarded a postdoctoral fellowship. S. Gupta and M. Igoillo-Esteve contributed equally to this work.

\section{References}

[1] M. P. Barrett, R. J. S. Burchmore, A. Stich et al., "The trypanosomiases," The Lancet, vol. 362, no. 9394, pp. 14691480, 2003.

[2] S. H. Funayama, S. Funayama, I. Y. Ito, and L. A. Veiga, “Trypanosoma cruzi: kinetic properties of glucose-6-phosphate dehydrogenase," Experimental Parasitology, vol. 43, no. 2, pp. 376-381, 1977.

[3] D. Barry, R. McCulloch, J. Mottram, and A. Acosta-Serrano, Trypanosomes: After the Genome, Horizon Bioscience, Norfolk, UK, 2007.

[4] A. Prata, "Clinical and epidemiological aspects of Chagas disease," Lancet Infectious Diseases, vol. 1, no. 2, pp. 92-100, 2001.

[5] K. M. Tyler and D. M. Engman, "The life cycle of Trypanosoma cruzi revisited," International Journal for Parasitology, vol. 31, no. 5-6, pp. 472-481, 2001.

[6] B. A. Burleigh and A. M. Woolsey, "Cell signalling and Trypanosoma cruzi invasion," Cellular Microbiology, vol. 4, no. 11, pp. 701-711, 2002.

[7] P. J. Myler and N. Fasel, Leishmania: After the Genome, Caister Academic Press, Norfolk, UK, 2008.

[8] J. C. Dujardin, D. González-Pacanowska, S. L. Croft, O. F. Olesen, and G. F. Späth, "Collaborative actions in antitrypanosomatid chemotherapy with partners from disease endemic areas," Trends in Parasitology, vol. 26, pp. 395-403, 2010.

[9] S. Nwaka and A. Hudson, "Innovative lead discovery strategies for tropical diseases," Nature Reviews Drug Discovery, vol. 5, no. 11, pp. 941-955, 2006.

[10] V. Delespaux and H. P. de Koning, "Drugs and drug resistance in African trypanosomiasis,” Drug Resistance Updates, vol. 10, no. 1-2, pp. 30-50, 2007.

[11] C. L. M. J. Verlinde, V. Hannaert, C. Blonski et al., "Glycolysis as a target for the design of new anti-trypanosome drugs," Drug Resistance Updates, vol. 4, no. 1, pp. 50-65, 2001.

[12] T. K. Smith and P. Bütikofer, "Lipid metabolism in Trypanosoma brucei," Molecular and Biochemical Parasitology, vol. 172, no. 2, pp. 66-79, 2010.

[13] N. Galland and P. A. M. Michels, "Comparison of the peroxisomal matrix protein import system of different organisms. Exploration of possibilities for developing inhibitors of the import system of trypanosomatids for anti-parasite chemotherapy," European Journal of Cell Biology, vol. 89, pp. 621-637, 2010.

[14] F. Agüero, B. Al-Lazikani, M. Aslett et al., "Genomic-scale prioritization of drug targets: the TDR Targets database," Nature Reviews Drug Discovery, vol. 7, no. 11, pp. 900-907, 2008.

[15] G. J. Crowther, D. Shanmugam, S. J. Carmona et al., "Identification of attractive drug targets in neglected- disease pathogens using an in silico approach," PLoS Neglected Tropical Diseases, vol. 4, no. 8, article e804, 2010.

[16] M. P. Barrett, "The pentose phosphate pathway and parasitic protozoa," Parasitology Today, vol. 13, no. 1, pp. 11-16, 1997.
[17] N. J. Kruger and A. Von Schaewen, "The oxidative pentose phosphate pathway: structure and organisation," Current Opinion in Plant Biology, vol. 6, no. 3, pp. 236-246, 2003.

[18] M. A. Rosemeyer, "The biochemistry of glucose-6-phosphate dehydrogenase, 6-phosphogluconate dehydrogenase and glutathione reductase," Cell Biochemistry and Function, vol. 5, no. 2, pp. 79-95, 1987.

[19] T. Wood, "Physiological functions of the pentose phosphate pathway," Cell Biochemistry and Function, vol. 4, no. 4, pp. 241-247, 1986.

[20] G. Ronquist and E. Theodorsson, "Inherited, non-spherocytic haemolysis due to deficiency of glucose-6-phosphate dehydrogenase," Scandinavian Journal of Clinical and Laboratory Investigation, vol. 67, no. 1, pp. 105-111, 2007.

[21] MD. Cappellini and G. Fiorelli, "Glucose-6-phosphate dehydrogenase deficiency," The Lancet, vol. 371, no. 9606, pp. 64$74,2008$.

[22] I. Raw, "Some aspects of carbohydrate metabolism of cultural forms of Trypanosoma cruzi," Revista do Instituto de Medicina Tropical de São Paulo, vol. 1, pp. 192-194, 1959.

[23] J. F. Ryley, "Studies on the metabolism of the protozoa. 9. Comparative metabolism of blood-stream and culture forms of Trypanosoma rhodesiense," The Biochemical Journal, vol. 85, pp. 211-223, 1962.

[24] R. Mancilla and C. Naquira, "Comparative metabolism of C14-glucose in two strains of Trypanosoma cruzi," The Journal of protozoology, vol. 11, pp. 509-513, 1964.

[25] R. Mancilla, C. Náquira, and C. Lanas, "Metabolism of glucose labelled with carbon-14 in Leishmania enriettii," Nature, vol. 206, no. 4979, pp. 27-28, 1965.

[26] R. Mancilla, C. Naquira, and C. Lanas, "Metabolism of glucose-C in Leishmania brasiliensis," Comparative Biochemistry and Physiology, vol. 28, no. 1, pp. 227-232, 1969.

[27] C. N. Cronín, D. P. Nolan, and H. P. Voorheis, "The enzymes of the classical pentose phosphate pathway display differential activities in procyclic and bloodstream forms of Trypanosoma brucei," FEBS Letters, vol. 244, no. 1, pp. 26-30, 1989.

[28] N. Heise and F. R. Opperdoes, "Purification, localisation and characterisation of glucose-6-phosphate dehydrogenase of Trypanosoma brucei," Molecular and Biochemical Parasitology, vol. 99, no. 1, pp. 21-32, 1999.

[29] F. Duffieux, J. Van Roy, P. A. M. Michels, and F, R. Opperdoes, "Molecular characterization of the first two enzymes of the pentose-phosphate pathway of Trypanosoma brucei: glucose-6-phosphate dehydrogenase and 6phosphogluconolactonase," Journal of Biological Chemistry, vol. 275, no. 36, pp. 27559-27565, 2000.

[30] F. R. Opperdoes and P. Borst, "Localization of non glycolytic enzymes in a microbody like organelle in Trypanosoma brucei: the glycosome," FEBS Letters, vol. 80, no. 2, pp. 360-364, 1977.

[31] C. Colasante, M. Ellis, T. Ruppert, and F. Voncken, "Comparative proteomics of glycosomes from bloodstream form and procyclic culture form Trypanosoma brucei brucei," Proteomics, vol. 6, no. 11, pp. 3275-3293, 2006.

[32] D. Vertommen, J. Van Roy, J. P. Szikora, M. H. Rider, P. A. M. Michels, and F. R. Opperdoes, "Differential expression of glycosomal and mitochondrial proteins in the two major lifecycle stages of Trypanosoma brucei," Molecular and Biochemical Parasitology, vol. 158, no. 2, pp. 189-201, 2008.

[33] P. A. M. Michels, F. Bringaud, M. Herman, and V. Hannaert, "Metabolic functions of glycosomes in trypanosomatids," Biochimica et Biophysica Acta, vol. 1763, no. 12, pp. 1463-1477, 2006. 
[34] O. Misset, O. J. M. Bos, and F. R. Opperdoes, "Glycolytic enzymes of Trypanosoma brucei. Simultaneous purification, intraglycosomal concentrations and physical properties," European Journal of Biochemistry, vol. 157, no. 2, pp. 441-453, 1986.

[35] F. R. Opperdoes, "Compartmentation of carbohydrate metabolism in trypanosomes," Annual Review of Microbiology, vol. 41, pp. 127-151, 1987.

[36] D. A. Maugeri and J. J. Cazzulo, "The pentose phosphate pathway in Trypanosoma cruzi," FEMS Microbiology Letters, vol. 234, no. 1, pp. 117-123, 2004.

[37] D. A. Maugeri, J. J. Cazzulo, R. J. S. Burchmore, M. P. Barrett, and P. O. J. Ogbunude, "Pentose phosphate metabolism in Leishmania mexicana," Molecular and Biochemical Parasitology, vol. 130, no. 2, pp. 117-125, 2003.

[38] N. J. Veitch, D. A. Maugeri, J. J. Cazzulo, Y. Lindqvist, and M. P. Barrett, "Transketolase from Leishmania mexicana has a dual subcellular localization,” Biochemical Journal, vol. 382, no. 2, pp. 759-767, 2004.

[39] F. R. Opperdoes and J. P. Szikora, "In silico prediction of the glycosomal enzymes of Leishmania major and trypanosomes," Molecular and Biochemical Parasitology, vol. 147, no. 2, pp. 193-206, 2006.

[40] . Funayama Sh., S. Funayama, I. Y. Ito, and L. A. Veiga, “Trypanosoma cruzi: kinetic properties of glucose-6-phosphate dehydrogenase," Experimental Parasitology, vol. 43, no. 2, pp. 376-381, 1977.

[41] J. A. Lupiañez, F. J. Adroher, A. M. Vargas, and A. Osuna, "Differential behaviour of glucose 6-phosphate dehydrogenase in two morphological forms of Trypanosoma cruzi," International Journal of Biochemistry, vol. 19, no. 11, pp. 1085-1089, 1987.

[42] V. Hannaert, E. Saavedra, F. Duffieux et al., "Plant-like traits associated with metabolism of Trypanosoma parasites," Proceedings of the National Academy of Sciences of the United States of America, vol. 100, no. 3, pp. 1067-1071, 2003.

[43] M. Igoillo-Esteve and J. J. Cazzulo, “The glucose-6-phosphate dehydrogenase from Trypanosoma cruzi: its role in the defense of the parasite against oxidative stress," Molecular and Biochemical Parasitology, vol. 149, no. 2, pp. 170-181, 2006.

[44] M. Igoillo-Esteve, D. Maugeri, A. L. Stern, P. Beluardi, and J. J. Cazzulo, "The pentose phosphate pathway in Trypanosoma cruzi: a potential target for the chemotherapy of Chagas disease," Anais da Academia Brasileira de Ciencias, vol. 79, no. 4, pp. 649-663, 2007.

[45] A. T. Cordeiro, O. H. Thiemann, and P. A. M. Michels, "Inhibition of Trypanosoma brucei glucose-6-phosphate dehydrogenase by human steroids and their effects on the viability of cultured parasites," Bioorganic and Medicinal Chemistry, vol. 17, no. 6, pp. 2483-2489, 2009.

[46] A. T. Cordeiro and O. H. Thiemann, "16-Bromoepiandrosterone, an activator of the mammalian immune system, inhibits glucose 6-phosphate dehydrogenase from Trypanosoma cruzi and is toxic to these parasites grown in culture," Bioorganic and Medicinal Chemistry, vol. 18, no. 13, pp. 4762-4768, 2010.

[47] I. Wenderoth, R. Scheibe, and A. Von Schaewen, "Identification of the cysteine residues involved in redox modification of plant plastidic glucose-6-phosphate dehydrogenase," Journal of Biological Chemistry, vol. 272, no. 43, pp. 26985-26990, 1997.

[48] C. Cséke, A. Balogh, and G. L. Farkas, "Redox modulation of glucose-6-P dehydrogenase in Anacystis nidulans and its 'uncoupling' by phage infection," FEBS Letters, vol. 126, no. 1, pp. 85-88, 1981.
[49] A. E. Leroux, D. A. Maugeri, F. R. Opperdoes, J. J. Cazzulo, and C. Nowicki, "Comparative studies on the biochemical properties of the malic enzymes from Trypanosoma cruzi and Trypanosoma brucei," FEMS Microbiology Letters, vol. 314, no. 1, pp. 25-33, 2011.

[50] P. Marks and J. Banks, "Inhibition of mammalian glucose6-phosphate dehydrogenase by steroids," Proceedings of the National Academy of Sciences of the United States of America, vol. 46, pp. 447-452, 1960.

[51] G. Gordon, M. C. Mackow, and H. R. Levy, "On the mechanism of interaction of steroids with human glucose 6-phosphate dehydrogenase," Archives of Biochemistry and Biophysics, vol. 318, no. 1, pp. 25-29, 1995.

[52] K. R. Rasmussen, M. J. Arrowood, and M. C. Healey, "Effectiveness of dehydroepiandrosterone in reduction of cryptosporidial activity in immunosuppressed rats," Antimicrobial Agents and Chemotherapy, vol. 36, no. 1, pp. 220-222, 1992.

[53] D. Freilich, S. Ferris, M. Wallace et al., "16 $\alpha$-bromoepiandrosterone, a dehydroepiandrosterone (DHEA) analogue, inhibits Plasmodium falciparum and Plasmodium berghei growth," American Journal of Tropical Medicine and Hygiene, vol. 63, no. 5-6, pp. 280-283, 2000.

[54] J. Morales-Montor, S. Baig, R. Mitchell, K. Deway, C. HallalCalleros, and R. T. Damian, "Immunoendocrine interactions during chronic cysticercosis determine male mouse feminization: role of IL-6," Journal of Immunology, vol. 167, no. 8, pp. 4527-4533, 2001.

[55] C. D. Dos Santos, M. P. Alonso Toldo, and J. C. Do Prado, "Trypanosoma cruzi: the effects of dehydroepiandrosterone (DHEA) treatment during experimental infection," Acta Tropica, vol. 95, no. 2, pp. 109-115, 2005.

[56] J. C. Carrero, C. Cervantes, N. Moreno-Mendoza, E. Saavedra, J. Morales-Montor, and J. P. Laclette, "Dehydroepiandrosterone decreases while cortisol increases in vitro growth and viability of Entamoeba histolytica," Microbes and Infection, vol. 8, no. 2, pp. 323-331, 2006.

[57] J. A. Vargas-Villavicencio, C. Larralde, and J. Morales-Montor, "Treatment with dehydroepiandrosterone in vivo and in vitro inhibits reproduction, growth and viability of Taenia crassiceps metacestodes," International Journal for Parasitology, vol. 38, no. 7, pp. 775-781, 2008.

[58] K. P. Luna, I. P. Hernández, C. M. Rueda, M. M. Zorro, S. L. Croft, and P. Escobar, "In vitro susceptibility of Trypanosoma cruzi strains from Santander, Colombia, to hexadecylphosphocholine (miltefosine), nifurtimox and benznidazole," Biomedica, vol. 29, no. 3, pp. 448-455, 2009.

[59] S. Gupta, A. T. Cordeiro, and P. A. M. Michels, "Glucose6-phosphate dehydrogenase is the targetfor the trypanocidal action of human steroids," Molecular and Biochemical Parasitology, vol. 176, no. 2, pp. 112-115, 2011.

[60] A. Cornish-Bowden, "Why is uncompetitive inhibition so rare? A possible explanation, with implications for the design of drugs and pesticides," FEBS Letters, vol. 203, no. 1, pp. 3-6, 1986. 

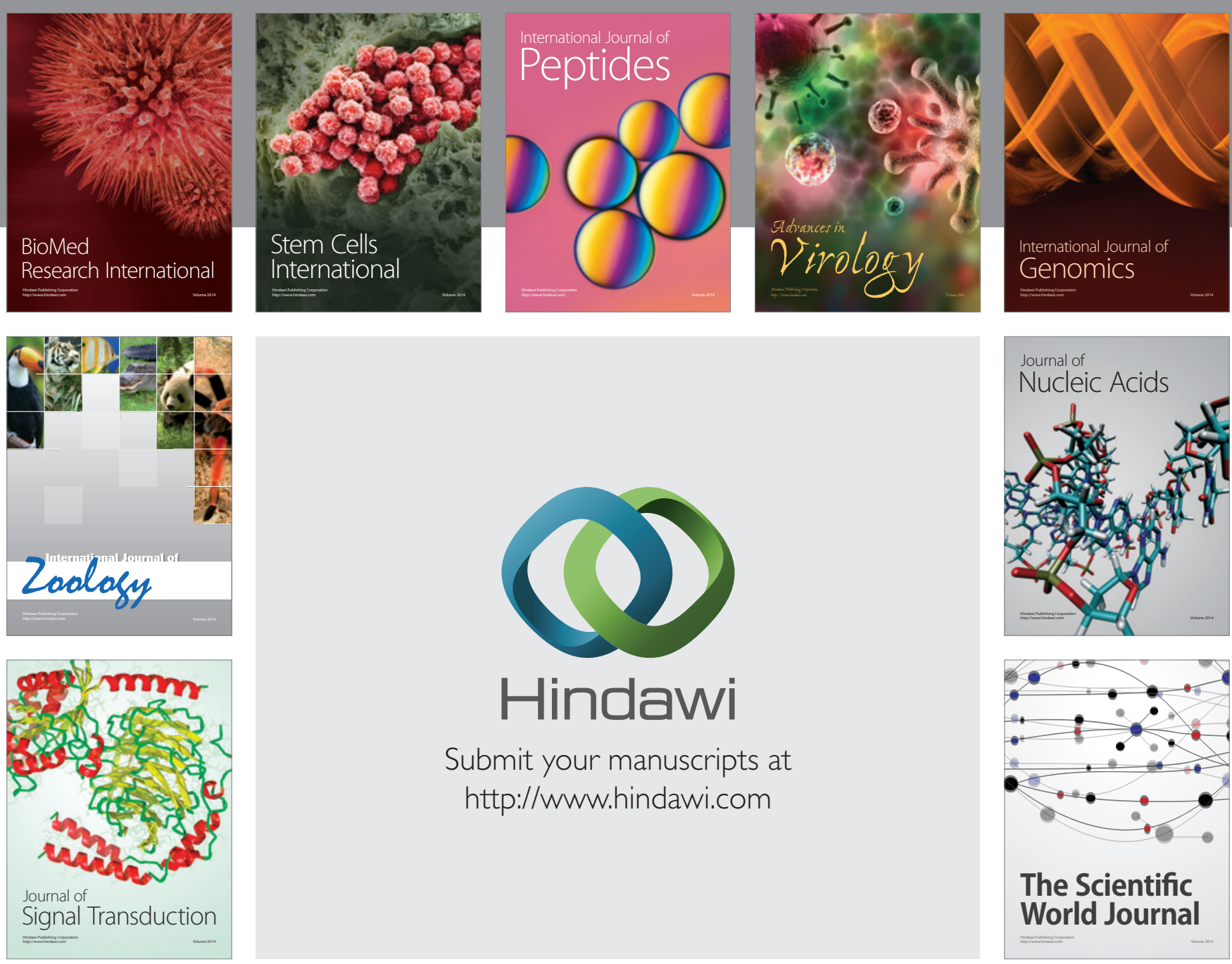

Submit your manuscripts at

http://www.hindawi.com
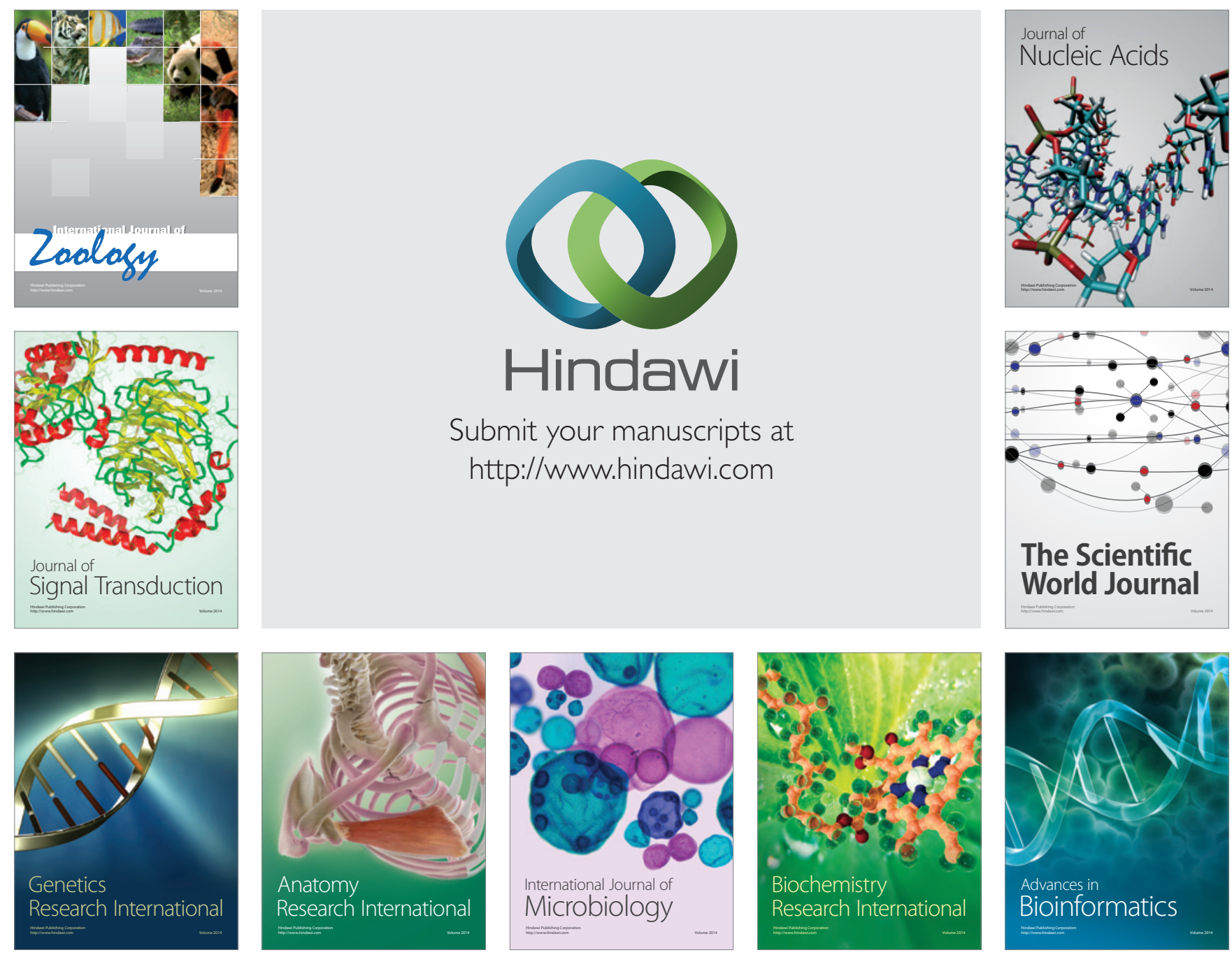

The Scientific World Journal
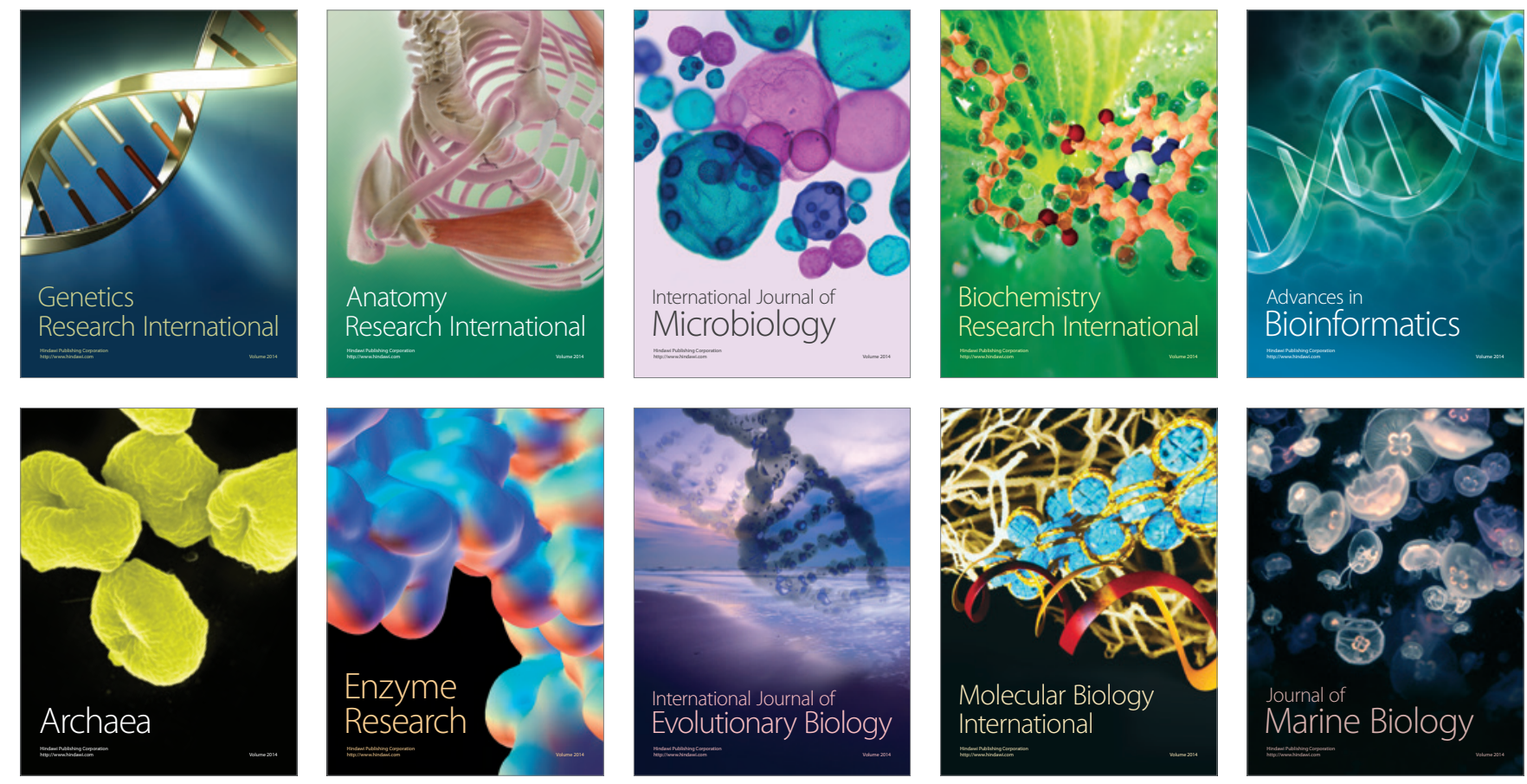